\title{
A Meta-Analysis of Brain-Derived Neurotrophic Factor Effects on Brain Volume in Schizophrenia: Genotype and Serum Levels
}

\author{
Anthony O. Ahmed ${ }^{\mathrm{a}}$ Samantha Kramer ${ }^{\mathrm{b}}$ Naama Hofman ${ }^{\mathrm{c}}$ John Flynn ${ }^{\mathrm{d}}$ \\ Marie Hansen ${ }^{d}$ Victoria Martin ${ }^{e}$ Anilkumar Pillai ${ }^{f}$ Peter F. Buckleyg \\ aDepartment of Psychiatry, Weill Cornell Medicine, White Plains, NY, USA; ${ }^{b}$ Department of Psychology, Long \\ Island University Post, New York, NY, USA; 'Department of Psychology, St. John's University, New York, NY, USA; \\ dDepartment of Psychology, Long Island University Brooklyn, New York, NY, USA; 'Department of Psychology, City \\ University of New York, New York, NY, USA; fDepartment of Psychiatry and Health Behavior, Augusta University, \\ Augusta, GA, USA; 'Department of Psychiatry, Virginia Commonwealth University, Richmond, VA, USA
}

\section{Keywords}

Brain-derived neurotrophic factor · Brain volume · rs6265 ·

Schizophrenia $\cdot$ Val66Met

\begin{abstract}
Aim: The Val66Met single-nucleotide polymorphism (SNP) on the BDNF gene has established pleiotropic effects on schizophrenia incidence and morphologic alterations in the illness. The effects of brain-derived neurotrophic factor (BDNF) on brain volume measurements are however mixed seeming to be less established for most brain regions. The current meta-analytic review examined (1) the association of the Val66Met SNP and brain volume alterations in schizophrenia by comparing Met allele carriers to Val/Val homozygotes and (2) the association of serum BDNF with brain volume measurements. Method: Studies included in the metaanalyses were identified through an electronic search of PubMed and PsycInfo (via EBSCO) for English language publications from January 2000 through December 2017. Included studies had conducted a genotyping procedure of Val66 Met or obtained assays of serum BDNF and obtained brain volume data in patients with psychotic disorders. Nonhuman studies were excluded. Results: Study 1 which included
\end{abstract}

karger@karger.com www.karger.com/nps

Karger $\stackrel{\text { ' }}{5}$

GOPEN ACCESS
C 2021 The Author(s).

Published by S. Karger AG, Basel

This is an Open Access article licensed under the Creative Commons Attribution-NonCommercial-4.0 International License (CC BY-NC) (http://www.karger.com/Services/OpenAccessLicense), applicable to the online version of the article only. Usage and distribution for commercial purposes requires written permission.
52 comparisons of Met carriers and $\mathrm{Val} / \mathrm{Val}$ homozygotes found evidence of lower right and left hippocampal volumes among Met allele carriers with schizophrenia. Frontal measurements, while also lower among Met carriers, did not achieve statistical significance. Study 2 which included 7 examinations of the correlation between serum BDNF and brain volume found significant associations between serum BDNF levels and right and left hippocampal volume with lower BDNF corresponding to lower volumes. Discussion: The meta-analyses provided evidence of associations between brain volume alterations in schizophrenia and variations on the Val66Met SNP and serum BDNF. Given the limited number of studies, it remains unclear if BDNF effects are global or regionally specific. @ 2021 The Author(s).

Published by S. Karger AG, Basel

\section{Introduction}

Several neuroimaging studies have shown that schizophrenia is characterized by alterations in brain morphology relative to controls $[1,2]$. These include enlarged ventricles and volumetric reductions within the frontal, temporal, occipital, parietal, and subcortical regions $[3,4]$. 
Volume losses in the dorsolateral prefrontal region, hippocampus, and amygdala have been linked to positive and negative symptoms as well as cognitive deficits [5-8]. The critical role of neurotrophins in regulating neurogenesis, synaptogenesis, and synaptic connectivity in several brain regions raises questions about whether alterations in their levels - also established in schizophrenia might explain volumetric alterations in the illness [9]. One such neurotrophin is the brain-derived neurotrophic factor (BDNF) which has been heavily studied in schizophrenia and other psychiatric illness [10-14]. Using postmortem samples, alterations in the intracellular signaling of BDNF have been demonstrated in some of the aforementioned brain regions including frontal, temporal, parietal, and occipital regions in people with schizophrenia relative to controls $[15,16]$. In particular, attention has been paid to aberrations in BDNF secretion and trafficking in the hippocampus where BDNF signaling has been found to be reduced in schizophrenia [17].

BDNF activity in the central nervous system appears to be related to a host of genetic influences, the most heavily studied being the Val66Met polymorphism on the $B D N F$ gene. Studies show associations between this single-nucleotide polymorphism (SNP) and brain volume alterations in people with schizophrenia. In particular, studies show that relative to $\mathrm{Val} / \mathrm{Val}$ homozygotes, carriers of the Met allele demonstrate (1) lower total brain volume; (2) greater reductions in frontal, temporal, and occipital regions; and (3) greater reductions in hippocampal volume [18-20]. The association of the Met allele with brain volume reductions in schizophrenia is mixed, however, seeming to be less established in some brain regions and measurements than others [21]. Moreover, the role of the BDNF protein may also be particular to certain regions. There is, for example, greater published evidence of BDNF transcription and translation activity in the hippocampus relative to other brain regions [22-24].

It is well established that the Val66Met SNP predicts the extent of BDNF intracellular traffic with lower BDNF signaling among Met allele carriers $[25,26]$. Clinical translational studies of BDNF in people with schizophrenia have mostly indexed BDNF levels using measures of peripheral BDNF (e.g., serum, plasma, and CSF). Studies of the association of Val66Met with peripheral BDNF have been mixed. Two studies in people with schizophrenia found that carriers of the Met allele demonstrated lower levels of peripheral BDNF [26, 27]. In contrast, other studies found no differences between Met carriers and $\mathrm{Val} / \mathrm{Val}$ homozygotes in peripheral BDNF measured in plasma and serum $[28,29]$. Other studies have exam- ined and found an association of BDNF signaling measured in brain tissue with serum BDNF levels [30-32]. These studies suggest that peripheral BDNF levels may reflect BDNF activity in the brain. This assertion - reasonable given that BDNF has been shown to cross the blood-brain barrier - is however tempered by the fact that blood-based BDNF is impacted by several other peripheral sources such as physical health, lifestyle choices, and platelet concentrations of BDNF [33-36]. Notwithstanding, most comparisons of people with schizophrenia with healthy controls have shown that the peripheral levels of BDNF are reduced in people with schizophrenia $[11,13,37]$. This finding appears independent of illness stage or the media of BDNF measurement (plasma, serum, or CSF).

In summary, the Val66Met SNP impacts BDNF mRNA activity in several brain regions and contributes to reduced cortical volume in schizophrenia, but findings of effects are equivocal in most brain regions investigated [21-24]. Reduced peripheral (serum) concentrations of BDNF in schizophrenia may reflect a reduction in brainbased BDNF. It is however unclear if peripheral BDNF as measured in the serum can robustly capture BDNF associations with reduced brain volume and thereby establish serum-based BDNF as an alternative means of investigating BDNF effects on cortical changes. A meta-analytic investigation could illuminate the size, consistency, and regional specificity of both genetic and serum effects on brain volume in schizophrenia. The literature on BDNF and brain volume has often segregated Val66Met and serum BDNF studies. Therefore, BDNF effects can be distilled into 2 reviews - first, on the association of the Val66Met SNP with brain volume reductions in schizophrenia and then the association of serum BDNF with cortical alterations.

The current study is a meta-analytic investigation of the association of BDNF with brain volume alterations in people with schizophrenia. Study 1 examined the association between the BDNF Val66Met SNP and brain volume alterations across several brain regions by comparing patients carrying the Met allele to $\mathrm{Val} / \mathrm{Val}$ homozygotes. Next, study 2 examined the association of serum BDNF levels with brain volume across several studies. The study 2 meta-analysis combines the results of correlational studies of BDNF and brain volume measures. It was predicted that carriers of the Met allele would demonstrate greater reductions in brain volume structures as well as larger ventricles. It was also predicted that there would be an inverse association between serum BDNF levels and brain volume reductions. 
Fig. 1. Consort diagram depicting the search strategy used for identifying studies included in the meta-analyses. BDNF, brain-derived neurotrophic factor.

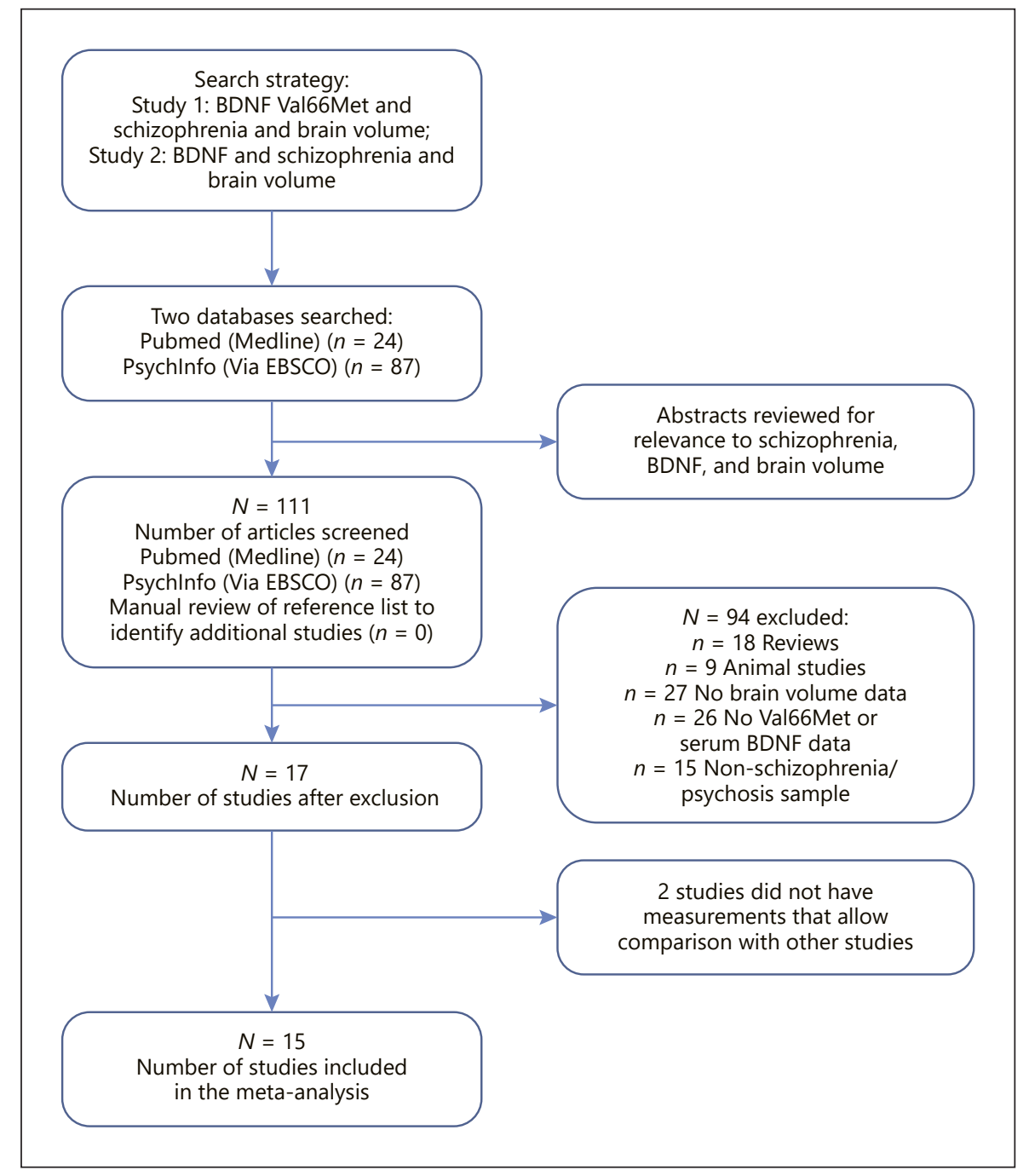

\section{Methods}

Search Strategy and Inclusion Criteria

Studies included in the meta-analyses were identified through an electronic search of PubMed and PsycInfo (via EBSCO) for English language publications from January 2000 through December 2019. The search strategy and reporting followed recommended PRISMA guidelines. The following search terms were used in each database: "BDNF Val66Met" AND "BDNF" or "Brain-Derived Neurotrophic Factor" AND "schizophrenia" AND "MRI” or "brain volume." Further, the reference list of articles obtained from the search was perused to identify additional articles. Figure 1 depicts the flowchart of the search strategy and included studies in the meta-analyses.

Two investigators reviewed titles and abstracts of citations identified through the database review to adjudge inclusion in the meta-analyses. There were no disagreements about studies that were ultimately included in the review. Studies meeting the following criteria were included: studies had obtained data from human participants, conducted a genotyping procedure of Val66Met or obtained assays of serum BDNF, participants had a diagnosis of a psychotic disorder, obtained brain volume data of either the whole brain or specific brain regions or structures, and obtained data needed for computing effect size differences between genotype groups including means and standard deviations (SDs), or correlations between serum BDNF and a brain volume measurement. For studies of the association of BDNF levels with brain volume, only those that obtained serum BDNF were included in the review. The Val66Met SNP was treated as a binary variable with both Met/Met homozygotes and Val/Met heterozygotes classified as a group of Met carriers to be compared to $\mathrm{Val} / \mathrm{Val}$ homozygotes.

\section{Data Extraction and Categorization}

Each study was labeled and indicated by the author and year of publication. For the Val66Met analysis, the sample size of each genotype subgroup, gender distribution, age, country, and education years of the sample were extracted from each study. The genotyping method, MRI measurement technique, and the mean and SD for each genotype group's brain volume measurement were also extracted from each study. For serum BDNF studies, 
BDNF correlations with examined brain regions were extracted from each study. Brain volume measurements in each study were classified based on the region or structure examined. The main structure/regions were frontal lobe, temporal lobe, right hippocampus, left hippocampus, and lateral ventricles.

\section{Data Analysis}

The extracted data were recorded on Microsoft Excel, and analyses were completed using the MIX 2.0 meta-analysis package [38]. MIX 2.0 interfaces with Microsoft Excel for the computation and creation of meta-analytic plots using a Visual Basic programming language. Effect size differences between Met carriers and $\mathrm{Val} / \mathrm{Val}$ homozygotes on brain volume were computed using the means, SDs, and sample sizes, and results reporting followed established PRISMA guidelines [39, 40]. Hedge's $g$ was used as the preferred effect size estimator over other estimators such as Cohen's $d$ because it produces less biased estimates when sample sizes are small and variance equality assumptions are violated [39]. Positive Hedge's $g$ values indicate that the Met allele carriers had greater volume reductions than Val/Val homozygotes. Pearson zero-order correlations between BDNF levels and brain volume measures served as effect size estimates in studies of peripheral BDNF. The mean effect size estimates were weighted for study sample size and variance, and a 95\% confidence interval (CI) to assess stability was obtained. The statistical significance of obtained effect size estimates, the heterogeneity of study effect sizes, and inconsistency across studies were all evaluated using the $Z$ statistic, Cochran's $Q$ statistic, and the $I^{2}$ metric, respectively. $I^{2}$ values range from 0 to $100 \%$ with 25,50 , and $75 \%$ designated as thresholds for low, moderate, and high heterogeneity [41]. Each meta-analysis was conducted assuming random effects. Corrections for multiple comparisons were applied to $Z$ statistic $p$ values using the Benjamini-Hochberg method with a false discovery rate set at $5 \%[42]$.

Synthesis forest plots were produced to represent effect sizes obtained from individual studies and an average effect size. Forest plots also served to depict the relative performance of both genotype groups or the size of the average BDNF-brain volume correlation. Sensitivity analyses were carried out to determine the degree to which an individual study biases the average effect size. Sensitivity analysis involves removing one study at a time and recomputing the average effect size. Visual inspections of selectivity funnel plots were used to assess publication bias - the propensity for journals to publish only studies that reject the null hypothesis. Selectivity funnel plots depict the study effect sizes (on the $x$-axis) against standard errors ( $y$-axis). Publication bias is usually indicated by an asymmetrical distribution of standard errors, whereas the absence of publication bias is usually indicated by a "funnel" distribution of standard errors [43]. Cases of publication bias were corrected for using the "trim and fill" method to produce an adjusted effect size estimate.

\section{Results}

\section{Study Search Results}

Figure 1 depicts the literature search strategy and the number of articles retrieved at each step. The initial search resulted in 111 articles which were subsequently screened to determine if they met the study inclusion criteria. Of the initial studies retrieved from PubMed and PsycInfo (EBSCOhost), 9 animal studies, 18 reviews, and 27 studies not including brain volume data were excluded from the analysis. An additional 26 articles that did not include Val66Met or serum BDNF data and 15 articles that did not report data from a nonschizophrenia/psychosis sample were excluded. The authors of studies that met inclusion criteria for the meta-analysis but for which the information needed to calculate effect sizes was not available in the original article were contacted to obtain the needed data. Studies from the same research team were examined carefully to ensure they did not use overlapping samples. Within individual studies however, when brain volume data were obtained in multiple regions, associations with each individual region were obtained within the same sample. It was further decided a priori that if an article reported on multiple independent samples, each sample would be treated as an independent study.

Study 1, which compared Met allele carriers and Val/ Val homozygotes on brain volume measures, included a total of 10 studies. The study 2 analysis of correlations between serum BDNF and brain volume included 5 studies. Retrieved studies obtained data from people with schizophrenia, schizoaffective disorder, or unspecified psychotic disorder.

\section{Study 1: The Association of Val66Met with Brain \\ Volume Measurements}

Table 1 summarizes the characteristics of all of the studies [18-21, 27, 44-48] that were included in the comparisons of genotype groups including gender distribution, age, education, genotype frequencies, and the imaging technique used in the study. In this meta-analysis, 52 comparisons of Met allele carriers and Val/Val homozygotes on brain volume variables were obtained from 10 independent studies. These comparisons included 926 people with an average age of 29.3 years. 401 participants were Met allele carriers, whereas 525 participants were Val/Val homozygotes. Table 2 presents effect size differences between Met carriers and Val/Val homozygotes by brain structure.

\section{Frontal Volume}

Frontal volume yielded 3 studies [19, 20, 48]. However, the 2 Ho and colleagues' $[19,20]$ studies measured overall frontal volume, and the Takahashi and colleagues' [48] study measured 12 individual structures in the frontal region in the same sample. We aggregated all of the frontal 


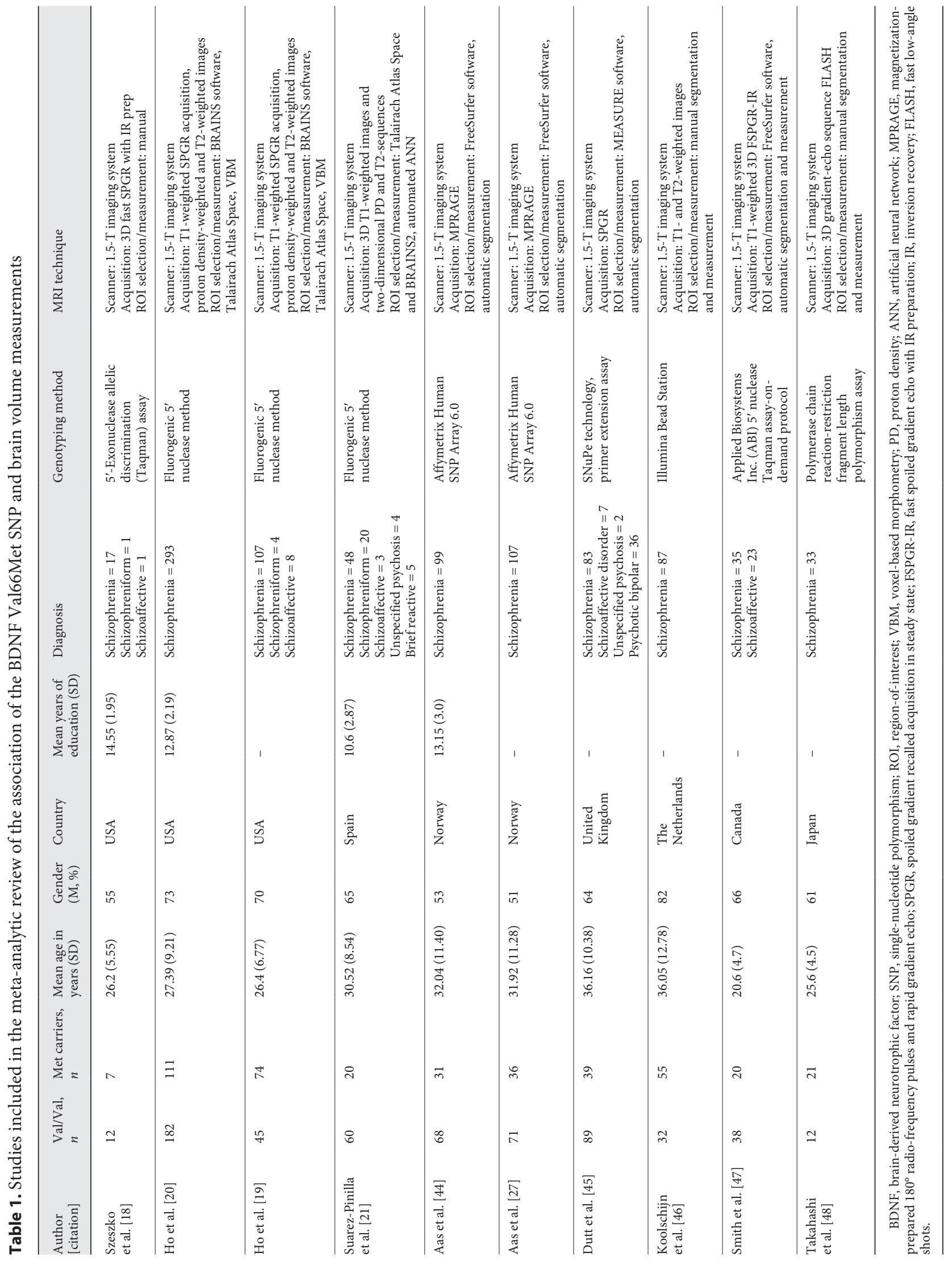




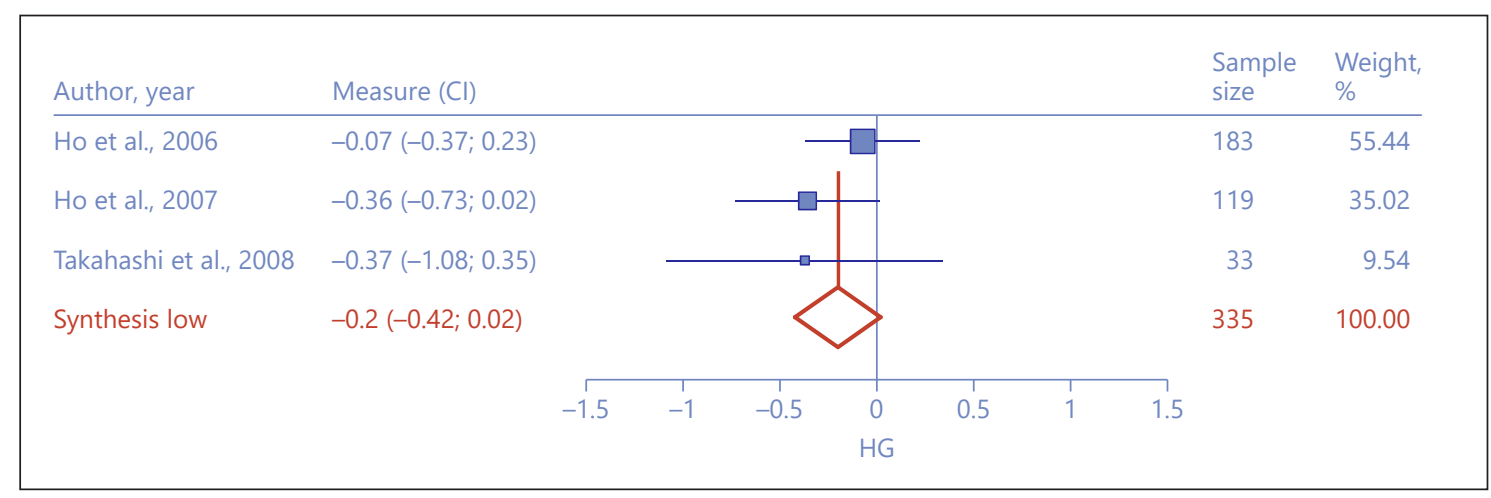

Fig. 2. Forest plot depicting effect size differences between Met allele carriers and Val/Val homozygotes on frontal volume measurements.

Table 2. Effect size differences between Met carriers and Val/Val homozygotes on brain volume measurements

\begin{tabular}{lllllllllll}
\hline Volume measurement & $\begin{array}{l}\text { Samples, } \\
n\end{array}$ & $\begin{array}{l}\text { Val, } \\
n\end{array}$ & $\begin{array}{l}\text { Met, } \\
n\end{array}$ & $\begin{array}{l}\text { Hedge's g, } \\
\text { hg }\end{array}$ & 95\% CI & $Z$ & $p$ value & $q^{*}$ \\
(corrected) & Q-test & \\
\hline Frontal & 3 & 129 & 206 & -0.20 & -0.42 to 0.02 & -1.77 & 0.08 & 0.11 & $Q=1.62, p=0.44$ \\
Right hippocampus & 5 & 236 & 162 & -0.44 & -0.79 to -0.09 & -2.50 & 0.01 & 0.02 & $Q=9.6, p=0.05$ \\
Left hippocampus & 5 & 236 & 162 & -0.31 & -0.52 to -0.10 & -2.87 & $<0.01$ & 0.0004 & $Q=2.58, p=0.63$ \\
Lateral ventricles & 4 & 346 & 193 & 0.20 & -0.32 to 0.71 & 0.75 & 0.46 & 0.46 & $Q=18.08, p<0.001$ \\
\hline
\end{tabular}

* $q$ Benjamini-Hochberg $p$ value with a false discovery rate set at 0.05 .

measurements in order to approximate an index of frontal volume in this single study and decrease the number of separate comparisons in the meta-analysis. Although Met allele carriers demonstrated smaller frontal volume than $\mathrm{Val} / \mathrm{Val}$ homozygotes, the small differences did not achieve statistical significance $(\mathrm{hg}=-0.20,95 \% \mathrm{CI}=$ -0.42 to $0.02, Z=-1.77, p=0.08$, see Fig. 2 ). The effect sizes showed little heterogeneity across studies $\left(I^{2}=0 \%\right.$, $Q=1.62, p=0.44)$, but the exclusion sensitivity analysis showed that removal of the Ho et al. $[19,20]$ sample increased the aggregate effect size and made it significant (hg $=-0.36,95 \% \mathrm{CI}=-0.69$ to $-0.03, Z=-2.13, p=0.03$ ) [43]. The exclusion selectivity test was unremarkable.

\section{Right Hippocampus}

Analysis of the right hippocampal volume yielded 5 studies and 5 samples [18, 44-46, 48]. Met allele carriers demonstrated significantly lower right hippocampal volume (hg $=-0.44,95 \% \mathrm{CI}=-0.79$ to $-0.09, Z=-2.50, p=$ 0.01 ) than $\mathrm{Val} / \mathrm{Val}$ homozygotes (Fig. 3). The effect sizes showed moderate significant heterogeneity $\left(I^{2}=58 \%\right.$,
$Q=9.6, p=0.05)$. Removal of the Szeszko et al. [18] study decreased the effect size but did not change the significance of the effects (hg $=-0.28,95 \% \mathrm{CI}=-0.51$ to -0.04 , $Z=-2.27, p=0.02$ ). In contrast, exclusion of the Dutt and colleagues [45] study increased the effect size (hg $=-0.56$, $95 \% \mathrm{CI}=-0.88$ to $-0.24, Z=-3.46, p<0.01)$. The selectivity funnel plot suggested slight publication bias with an asymmetrical distribution of standard errors. The corrected effect size was hg $=-0.27(95 \% \mathrm{CI}=-0.47$ to $-0.07)$.

\section{Left Hippocampus}

The left hippocampus similarly yielded 5 studies and 5 samples [18, 44-46, 48]. Met allele carriers demonstrated significantly lower left hippocampal volume (hg $=-0.31$, $95 \% \mathrm{CI}=-0.52$ to $-0.10, Z=-2.87, p<0.01)$ than $\mathrm{Val} /$ Val homozygotes (Fig. 4). No heterogeneity was observed across study effect sizes $\left(I^{2}=0 \%, Q=2.58, p=0.63\right)$, and the exclusion sensitivity test showed no propensity for the aggregated effect size to change remarkably due to the exclusion of any study. The selectivity funnel plot and Eg- 


\begin{tabular}{|c|c|c|c|c|c|c|c|c|c|c|}
\hline Author, year & Measure $(\mathrm{Cl})$ & & & & & & & & $\begin{array}{l}\text { Sample } \\
\text { size }\end{array}$ & $\begin{array}{l}\text { Weight, } \\
\%\end{array}$ \\
\hline Aas et al., 2013 & $-0.41(-0.84 ; 0.02)$ & & & 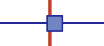 & & & & & 106 & 23.04 \\
\hline Dutt et al., 2009 & $0(-0.38 ; 0.38)$ & & & & & & & & 128 & 24.97 \\
\hline Koolschijn et al., 2010 & $-0.38(-0.82 ; 0.06)$ & & & e & & & & & 87 & 22.54 \\
\hline Szezsko et al., 2005 & $-1.16(-1.81 ;-0.5)$ & 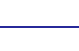 & & & & & & & 44 & 15.57 \\
\hline Takahashi et al., 2008 & $-0.57(-1.29 ; 0.15)$ & & & & & & & & 33 & 13.88 \\
\hline \multirow[t]{3}{*}{ Synthesis low } & $-0.44(-0.79 ;-0.09)$ & & & & & & & & 398 & 100.00 \\
\hline & -2 & -1.5 & -1 & -0.5 & 0 & 0.5 & 1 & 1.5 & & \\
\hline & & & & HG & & & & & & \\
\hline
\end{tabular}

Fig. 3. Forest plot depicting effect size differences between Met allele carriers and Val/Val homozygotes on right hippocampal volume.

\begin{tabular}{|c|c|c|c|c|c|c|c|c|c|}
\hline Author, year & Measure $(\mathrm{Cl})$ & & & & & & & $\begin{array}{l}\text { Sample } \\
\text { size }\end{array}$ & $\begin{array}{l}\text { Weight, } \\
\%\end{array}$ \\
\hline Aas et al., 2013 & $-0.38(-0.81 ; 0.04)$ & & $\square$ & & & & & 106 & 24.74 \\
\hline Dutt et al., 2009 & $-0.14(-0.51 ; 0.24)$ & & & 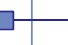 & & & & 128 & 31.59 \\
\hline Koolschijn et al., 2010 & $-0.27(-0.7 ; 0.17)$ & & & \begin{tabular}{l|l} 
\\
\end{tabular} & & & & 87 & 23.42 \\
\hline Szezsko et al., 2005 & $-0.72(-1.34 ;-0.09)$ & & & & & & & 44 & 11.45 \\
\hline Takahashi et al., 2008 & $-0.31(-1.02 ; 0.4)$ & & & & & & & 33 & 8.81 \\
\hline \multirow[t]{2}{*}{ Synthesis low } & $-0.31(-0.52 ;-0.1)$ & & & & & & & 398 & 100.00 \\
\hline & \multicolumn{6}{|c|}{$H G$} & 1.5 & & \\
\hline
\end{tabular}

Fig. 4. Forest plot depicting effect size differences between Met allele carriers and Val/Val homozygotes on left hippocampal volume.

ger's test showed no publication bias (Egger's test: $Z=0.5$, $p=0.61)$.

\section{Lateral Ventricles}

Analysis of ventricular size yielded 4 studies and 4 samples [19, 21, 44, 45]. Of note, the Aas et al. [44] study measured the right and left ventricles separately. The group means were collapsed in the meta-analysis to obtain an aggregate lateral ventricular size. Although Met allele carriers demonstrated larger ventricular size than $\mathrm{Val} / \mathrm{Val}$ homozygotes, the small differences did not achieve statistical significance $(\mathrm{hg}=0.20,95 \% \mathrm{CI}=-0.32$ to $0.71, Z=0.75, p=0.46$, Fig. 5). The effect sizes demonstrated large heterogeneity across studies $\left(I^{2}=83 \%, Q=\right.$ 18.08, $p<0.001)$. The exclusion sensitivity analysis showed that exclusion of the Suárez-Pinilla et al. [21] study increased the effect size but did not impact statistical significance (hg $=0.40,95 \% \mathrm{CI}=-0.10$ to $0.90, Z=$ $1.56, p=0.12)$.

\section{Study 2: Association between Serum BDNF and Brain Volume}

A second meta-analysis examined the correlation between serum BDNF and brain volume measurements. 


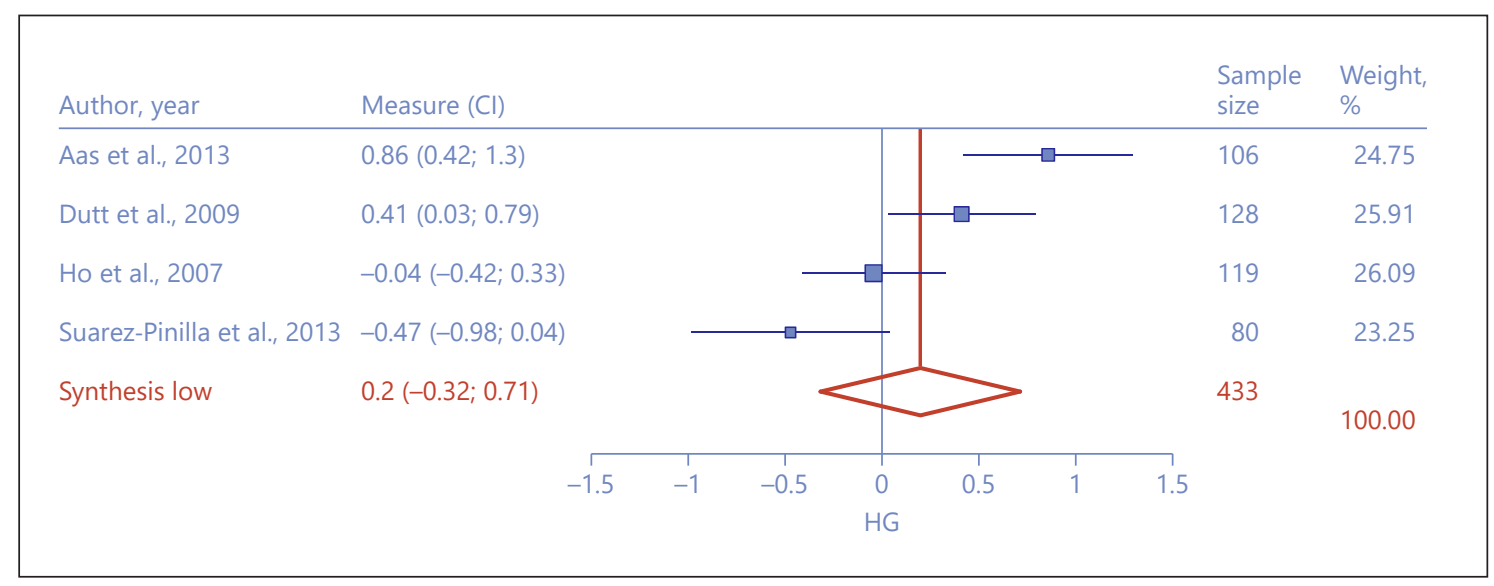

Fig. 5. Forest plot depicting effect size differences between Met allele carriers and Val/Val homozygotes on ventricular size.

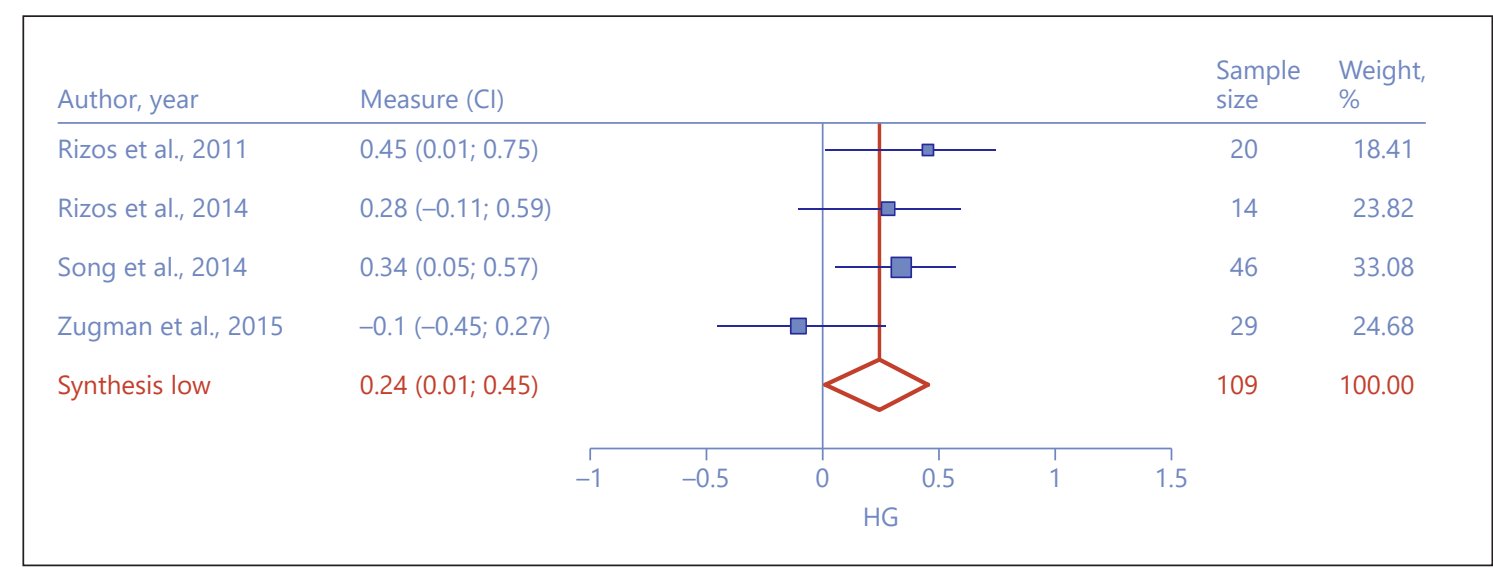

Fig. 6. Forest plot depicting the association of serum BDNF with right hippocampal volume.

This included 5 studies and 8 correlations of BDNF and a brain volume measurement [49-53]. Table 3 summarizes the sample characteristics of all of the studies that were included in the analysis. The analysis included 98 men and 60 women with an average age of 28.67 years. Four of the samples examined BDNF correlations with the right hippocampus and 4 examined correlations with the left hippocampus. One study examined correlations with the left and right amygdala and total cortical volume [49] and another examined insula thickness [53], but no other studies examined any nonhippocampal structure. Therefore, analyses were limited to the right and left hippocampus.

\section{Right Hippocampus}

Four studies obtained correlations between serum $\mathrm{BDNF}$ and the right hippocampus in 4 samples [49-52].
There was a small, statistically significant correlation between BDNF levels and the volume of the right hippocampus $(r=0.24,95 \% \mathrm{CI}=0.01-0.45, Z=2.05, p=0.04$, Fig. 6). The $Q$-test of effect size heterogeneity indicated no significant differences in correlations across studies $\left(I^{2}=36.23 \%, Q=4.70, p=0.19\right)$. However, the exclusion sensitivity test suggested that exclusion of the Zugman et al. [49] study increased the average correlation $(r=0.35$, $95 \% \mathrm{CI}=0.15-0.52, Z=3.32, p<0.01$ ). The selectivity funnel plot and Egger's test showed no evidence of publication bias (Egger's test: $Z=0.28, p=0.78$ ).

\section{Left Hippocampus}

Three studies with 3 independent samples similarly obtained correlations between BDNF and left hippocampal volume $[49,51,52]$. BDNF similarly showed a medi- 


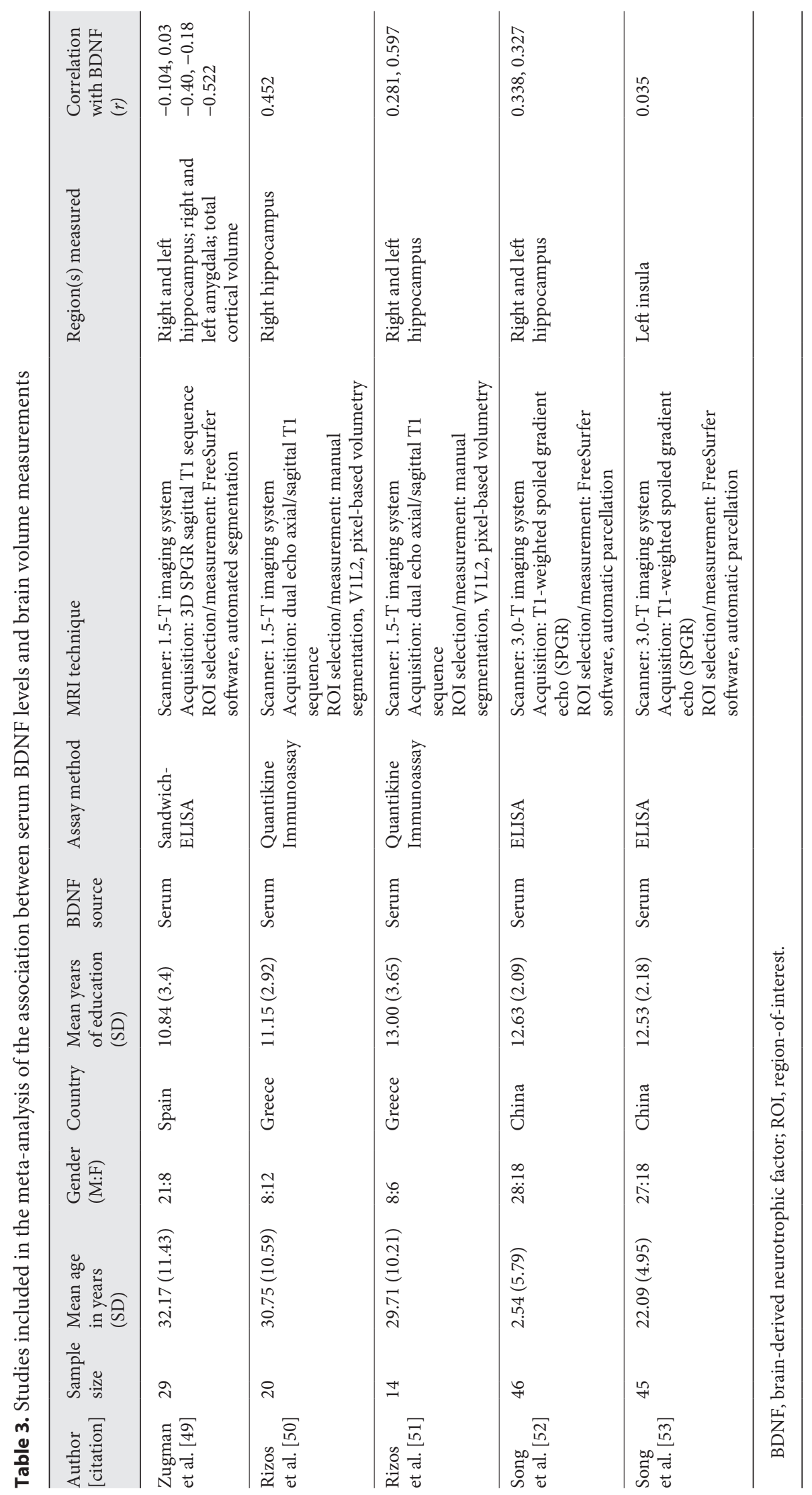




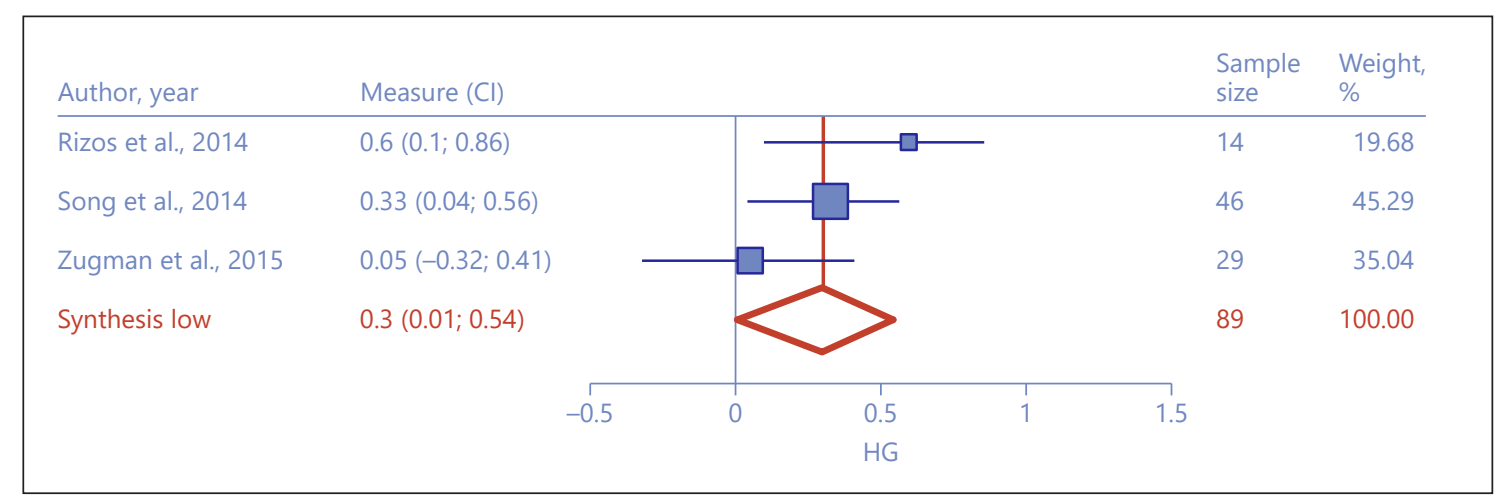

Fig. 7. Forest plot depicting the association of serum BDNF with left hippocampal volume.

um, significant correlation with the volume of the left hippocampus ( $r=0.30,95 \% \mathrm{CI}=0.01-0.54, Z=2.00, p=$ 0.046 , Fig. 7). The $Q$-test showed low, statistically nonsignificant differences in correlation effect size across studies $\left(I^{2}=40.26 \%, Q=3.35, p=0.19\right)$. The exclusion sensitivity test suggested that exclusion of the Zugman et al. [49] study slightly increased the average correlation $(r=$ $0.39,95 \% \mathrm{CI}=0.13-0.61, Z=2.87, p<0.01)$. The selectivity funnel plot and Egger's test showed no evidence of publication bias (Egger's test: $Z=0.51, p=0.60$ ).

\section{Discussion}

The current meta-analytic review examined the association between BDNF and brain volume in people with schizophrenia. Study 1 examined the association of the Val66Met allele with brain volume measurements by comparing Met allele carriers with $\mathrm{Val} / \mathrm{Val}$ homozygotes on brain volume measures. Study 2 further examined the role of BDNF on brain volume changes by examining the correlation between serum-based measurements of $\mathrm{BDNF}$ and brain volume. In all, the meta-analysis included 15 studies and 59 evaluations of the association of BDNF and a brain volume assessment. This included 52 comparisons of Met carriers and Val/Val homozygotes (study 1) and 7 examinations of the correlation between BDNF levels and brain volume (study 2). Study 1 provided evidence of significant differences between the genotype groups in the volume of the right and left hippocampus with lower volumes being apparent for Met allele carriers. However, frontal volume was also lower among Met allele carriers, and frontal differences just missed statistical significance in the meta-analysis. There were not enough published studies from independent research groups to ascertain genotype group differences in temporal, occipital, parietal, and amygdala volumes. In study 2 , data were only available to examine the correlation between serum BDNF and the left and right hippocampal volume. The studies produced a small, significant correlation between serum BDNF and right hippocampal volume and a medium, significant correlation between serum BDNF and left hippocampal volume.

The results of the current study are in slight contrast to results of previous meta-analyses that examined the association of Val66Met with brain volume in clinical and nonclinical samples [22-24]. The Harrisberger et al. [23] and the Molendijk et al. [24] meta-analyses reported smaller hippocampal volumes for Met allele carriers, but differences obtained were in the minimal range. Of note, their analyses included samples with various psychiatric diagnoses including depression, bipolar disorder, anxiety disorders, and schizophrenia. In contrast, the current study examined the effect of the Val66Met gene in schizophrenia samples alone. None of the previous meta-analyses examined the association of peripheral BDNF and brain volume measurements. In contrast, the current meta-analysis further supported a role for reduced BDNF in hippocampal volume reductions in schizophrenia by demonstrating an association. It may be that the Val$66 \mathrm{Met}$ SNP has a greater impact on brain volume reductions in schizophrenia relative to healthy controls and people with mood and anxiety disorders. It is also possible that the Val66Met effect on brain volume loss operates in synergy with variations in other risk genes with pleiotropic effects on schizophrenia risk and volume loss.

The current meta-analysis shows that the Val66Met SNP on the BDNF gene and serum BDNF levels are associated with the degree of schizophrenia-related volume losses in the hippocampus and frontal lobe. A putative 
causal mechanism could be that the Val-Met substitution attenuates activity-dependent BDNF packaging and transport in the central nervous system. Lower BDNF in turn contributes to reduced neurogenesis, synaptogenesis, and neuroplasticity most prominently in hippocampal and frontal regions. Remarkably, the effect of BDNF did not appear to be robust across all brain volume measurements. Effects on frontal structures were marginal, and effects on ventricular size were largely absent. Other existing brain volume measurements including temporal, parietal, occipital, amygdala, gray matter, white matter, and overall intracranial volume were poorly represented in the literature and could not be evaluated in relation to $\mathrm{BDNF}$. It is possible that BDNF signaling is most salient to hippocampal and frontal structure and function than other brain regions; however, the limited number of studies that have investigated other regions tempers this assertion. Notwithstanding, the small to medium range correlations between serum BDNF levels and left and right hippocampal volume are promising and may suggest at least similar correlations between brain-based BDNF signaling and volumetric measures.

The hippocampus is well established as the site for memory, visual-spatial abilities, and learning-dependent long-term potentiation, whereas the frontal regions regulate executive functions [54-56]. A previous meta-analysis obtained small associations between the BDNF Val66Met SNP and performance on visual and verbal learning cognitive tasks and small significant correlations between peripheral BDNF levels and performance on reasoning and problem-solving tasks [57]. Effects on other cognitive abilities in people with schizophrenia were, however, absent. These observations suggest that BDNF effects on hippocampal and frontal volume may exercise downstream effects on specific cognitive abilities influenced by these regions. Cognitive remediation, an intervention shown to improve cognitive functions in people with schizophrenia, has been separately studied in relation to increased serum BDNF and brain volume [58-64]. A demonstration that cognitive training-related improvements in brain volume measures are mediated by changes in serum BDNF levels would further support the assertion of BDNF effects on brain volume. Compounds shown to improve cognition and function in schizophrenia should be similarly investigated to determine if increased BDNF mediates treatment gains $[65,66]$.

Studies have shown that BDNF signaling is attenuated in people with schizophrenia relative to control samples - perhaps related to increased risk of the Met allele [13]. Reduced BDNF levels imply that its effects on neural

BDNF and Brain Volume in Schizophrenia survival processes such as neurogenesis, synaptogenesis, neurotransmission, and neuroplasticity are reduced in schizophrenia [67]. BDNF may therefore contribute to observed brain volume reductions in schizophrenia, especially in regions of significant BDNF function such as the hippocampal and frontal regions. It is likely, however, that BDNF works synergistically with other neurotrophins, growth factors, and immune response parameters that have similarly shown associations with frontal and hippocampal volume [68-70]. The current meta-analysis adds to existing evidence of the status of BDNF as a biomarker of schizophrenia with multiple pleiotropic effects including the incidence of the illness, predisposing factors, cognitive functions, and clinical status $[37,50,71-$ 81].

\section{Limitations}

The current study did not evaluate the role of demographic characteristics such as age, gender, race, and education. The overall number of studies available for inclusion in the meta-analysis was low, thereby limiting the extent to which these variables could be examined in relation to BDNF and brain volume. Studies have shown that there may be racial differences in the Val66Met SNP with an increased frequency of the Met allele among people of Asian descent. It is therefore unclear from the current review if such differences in allelic frequency impact BDNF signaling or brain volume. Several sociodemographic variables including older age, sex, and urbanicity, as well as lifestyle variables including nutrition, exercise, smoking status, and alcohol use, have been shown to influence serum BDNF levels $[33,35]$. The impacts of physical health and mood symptoms on serum BDNF have also been documented $[34,36]$. These variables, including several that may similarly impact brain volume such as medications, substance use, illness onset, comorbid illness, duration, and illness course, were not extensively documented in the individual studies and were not evaluated in the current meta-analysis [82].

As shown in Tables 1 and 3, studies used diverse genotyping or assaying methods. Genotyping methods may differ in their ability to distinguish Met allele carriers, and ELISA kits may differ in their ability to distinguish BDNF isoforms and accurately measure BDNF concentrations. Moreover, other technical issues may impact accurate measurement of BDNF levels including storage time of samples and the time of withdrawal [33]. These variables were often not documented in individual studies and could not be evaluated for their impact on peripheral BDNF levels. 
Most studies of peripheral BDNF in schizophrenia have examined blood serum concentrations (see Green et al. [13]). The studies included in the current meta-analysis similarly obtained BDNF levels from blood serum. However, the concentration of platelets in serum or plasma samples is also an important determinant of peripheral $\mathrm{BDNF}$ concentrations given that $\mathrm{BDNF}$ accumulates in platelets after it is synthesized in megakaryocytes [83, $84]$. It is unclear if any of the studies included in the metaanalysis corrected for the concentration of platelets in their samples. Platelet concentrations in media sources were moreover unavailable for us to include as a covariate in our analyses. It is known that BDNF obtained from the serum is often higher than that obtained from the plasma after the removal of blood platelets. Thus, the extent to which peripheral BDNF reflects CNS BDNF signaling remains in question.

\section{Conclusions}

The current meta-analytic study suggests that although effects are yet to be demonstrated across all brain regions, there are nontrivial associations between the Val66Met SNP and hippocampal volume and peripheral BDNF measured in the blood and hippocampal volume. The study also showed evidence of marginal effects on frontal volume measurement with Met allele carriers demonstrating greater volumetric reductions. It is noteworthy that the available number of studies and sample sizes obtained in individual studies were generally low which may inadvertently limit statistical power or overestimate overall effect sizes. Notwithstanding sample limitations and the need for adequately powered studies, the meta-analyses provide information about a potential risk factor for volumetric declines in schizophrenia.

\section{Statement of Ethics}

The study is exempt from ethics committee approval.

\section{Conflict of Interest Statement}

The authors have no conflicts of interest to disclose.

\section{Funding Sources}

The authors did not receive any funding.

\section{Author Contributions}

Anthony O. Ahmed and Samantha Kramer created the first draft of the manuscript. Naama Hofman and John Flynn compiled the literature needed for analyses and also helped with revising the manuscript. Marie Hansen and Victoria Martin participated in drafting the manuscript. Anilkumar Pillai and Peter F. Buckley helped with conceptualizing the project and revising drafts of the manuscript.

\section{References}

1 Bakhshi K, Chance SA. The neuropathology of schizophrenia: a selective review of past studies and emerging themes in brain structure and cytoarchitecture. Neuroscience. 2015;303:82-102.

2 Olabi B, Ellison-Wright I, McIntosh AM, Wood SJ, Bullmore E, Lawrie SM. Are there progressive brain changes in schizophrenia? A meta-analysis of structural magnetic resonance imaging studies. Biol Psychiatry. 2011; 70(1):88-96.

3 Ahmed AO, Buckley PF, Hanna M. Neuroimaging schizophrenia: a picture is worth a thousand words, but is it saying anything important? Curr Psychiatry Rep. 2013;15(3): 345.

4 Honea R, Crow TJ, Passingham D, Mackay CE. Regional deficits in brain volume in schizophrenia: a meta-analysis of voxel-based morphometry studies. Am J Psychiatry. 2005; 162(12):2233-45.
5 Czepielewski LS, Wang L, Gama CS, Barch DM. The relationship of intellectual functioning and cognitive performance to brain structure in schizophrenia. Schizophr Bull. 2017; 43(2):355-64

6 Lui S, Deng W, Huang X, Jiang L, Ma X, Chen $\mathrm{H}$, et al. Association of cerebral deficits with clinical symptoms in antipsychotic-naive first-episode schizophrenia: an optimized voxel-based morphometry and resting state functional connectivity study. Am J Psychiatry. 2009;166(2):196-205.

7 Suzuki M, Zhou SY, Hagino H, Niu L, Takahashi T, Kawasaki Y, et al. Morphological brain changes associated with Schneider's first-rank symptoms in schizophrenia: a MRI study. Psychol Med. 2005;35:549-60.

8 Veijola J, Guo JY, Moilanen JS, Jääskeläinen E, Miettunen J, Kyllönen M, et al. Longitudinal changes in total brain volume in schizophrenia: relation to symptom severity, cognition and antipsychotic medication. PLoS One. 2014;9(7):e101689.
9 Buckley PF, Mahadik S, Pillai A, Terry A. Neurotrophins and schizophrenia. Schizophr Res. 2007;94(1-3):1-11.

10 Autry AE, Monteggia LM. Brain-derived neurotrophic factor and neuropsychiatric disorders. Pharmacol Rev. 2012;64(2):238-58.

11 Buckley PF, Pillai A, Howell KR. Brain-derived neurotrophic factor: findings in schizophrenia. Curr Opin Psychiatry. 2011;24(2):122-7.

12 Durany N, Michel T, Zöchling R, Boissl KW Cruz-Sánchez FF, Riederer P, et al. Brain-derived neurotrophic factor and neurotrophin 3 in schizophrenic psychoses. Schizophr Res. 2001;52(1-2):79-86

13 Green MJ, Matheson SL, Shepherd A, Weickert CS, Carr VJ. Brain-derived neurotrophic factor levels in schizophrenia: a systematic review with meta-analysis. Mol Psychiatry. 2011;16(9):960-72.

14 Pandya CD, Kutiyanawalla A, Pillai A. BDNFTrkB signaling and neuroprotection in schizophrenia. Asian J Psychiatr. 2013;6(1): $22-8$. 
15 Ray MT, Shannon Weickert C, Webster MJ. Decreased BDNF and TrkB mRNA expression in multiple cortical areas of patients with schizophrenia and mood disorders. Transl Psychiatry. 2014;4:e389.

16 Weickert CS, Hyde TM, Lipska BK, Herman MM, Weinberger DR, Kleinman JE. Reduced brain-derived neurotrophic factor in prefrontal cortex of patients with schizophrenia. Mol Psychiatry. 2003;8(6):592-610.

17 Ray MT, Weickert CS, Wyatt E, Webster MJ. Decreased BDNF, trkB-TK+ and GAD67 mRNA expression in the hippocampus of individuals with schizophrenia and mood disorders. J Psychiatry Neurosci. 2011;36:195203.

18 Szeszko PR, Lipsky R, Mentschel C, Robinson D, Gunduz-Bruce H, Sevy S, et al. Brain-derived neurotrophic factor val66met polymorphism and volume of the hippocampal formation. Mol Psychiatry. 2005;10(7):631-6.

19 Ho BC, Andreasen NC, Dawson JD, Wassink TH. Association between brain-derived neurotrophic factor Val66Met gene polymorphism and progressive brain volume changes in schizophrenia. Am J Psychiatry. 2007; 164(12):1890-9.

20 Ho BC, Milev P, O'Leary DS, Librant A, Andreasen NC, Wassink TH. Cognitive and magnetic resonance imaging brain morphometric correlates of brain-derived neurotrophic factor Val66Met gene polymorphism in patients with schizophrenia and healthy volunteers. Arch Gen Psychiatry. 2006;63(7): $731-40$.

21 Suárez-Pinilla P, Roiz-Santiáñez R, de la Foz VO, Mata I, Fañanas L, Brambilla P, et al. BDNF Val66Met variants and brain volume changes in non-affective psychosis patients and healthy controls: a 3 year follow-up study. Prog Neuropsychopharmacol Biol Psychiatry. 2013;45:201-6.

22 Harrisberger F, Spalek K, Smieskova R, Schmidt A, Coynel D, Milnik A, et al. The association of the BDNF Val66Met polymorphism and the hippocampal volumes in healthy humans: a joint meta-analysis of published and new data. Neurosci Biobehav Rev. 2014;42:267-78.

23 Harrisberger F, Smieskova R, Schmidt A, Lenz C, Walter A, Wittfeld K, et al. BDNF Val66Met polymorphism and hippocampal volume in neuropsychiatric disorders: a systematic review and meta-analysis. Neurosci Biobehav Rev. 2015;55:107-18.

24 Molendijk ML, Bus BAA, Spinhoven P, Kaimatzoglou A, Oude Voshaar RC, Penninx $\mathrm{BW}$, et al. A systematic review and meta-analysis on the association between BDNF val(66) met and hippocampal volume: a genuine effect or a winners curse? Am J Med Genet B Neuropsychiatr Genet. 2012;159B:731-40.

25 Chiaruttini C, Vicario A, Li Z, Baj G, Braiuca $\mathrm{P}, \mathrm{Wu} \mathrm{Y}$, et al. Dendritic trafficking of BDNF mRNA is mediated by translin and blocked by the G196A (Val66Met) mutation. Proc Natl Acad Sci U S A. 2009;106(38):16481-6.
26 Zakharyan R, Boyajyan A. Brain-derived neurotrophic factor blood levels are decreased in schizophrenia patients and associate with rs6265 genotypes. Clin Biochem. 2014;47(12): 1052-5.

27 Aas M, Haukvik UK, Djurovic S, Tesli M, Athanasiu L, Bjella T, et al. Interplay between childhood trauma and BDNF val66met variants on blood BDNF mRNA levels and on hippocampus subfields volumes in schizophrenia spectrum and bipolar disorders. J Psychiatr Res. 2014;59:14-21.

28 Chen SL, Lee SY, Chang YH, Chen SH, Chu $\mathrm{CH}$, Wang TY, et al. The BDNF Val66Met polymorphism and plasma brain-derived neurotrophic factor levels in Han Chinese patients with bipolar disorder and schizophrenia. Prog Neuropsychopharmacol Biol Psychiatry. 2014;51:99-104.

29 Zhang XY, Chen DC, Xiu MH, Haile CN, Luo $\mathrm{X}, \mathrm{Xu} \mathrm{K}$, et al. Cognitive and serum BDNF correlates of BDNF Val66Met gene polymorphism in patients with schizophrenia and normal controls. Hum Genet. 2012;131(7): 1187-95.

30 Klein AB, Williamson R, Santini MA, Clemmensen C, Ettrup A, Rios M, et al. Blood BDNF concentrations reflect brain-tissue BDNF levels across species. Int J Neuropsychopharmacol. 2011;14(3):347-53.

31 Lang UE, Hellweg R, Seifert F, Schubert F, Gallinat J. Correlation between serum brainderived neurotrophic factor level and an in vivo marker of cortical integrity. Biol Psychiatry. 2007;62(5):530-5.

32 Sartorius A, Hellweg R, Litzke J, Vogt M, Dormann C, Vollmayr B, et al. Correlations and discrepancies between serum and brain tissue levels of neurotrophins after electroconvulsive treatment in rats. Pharmacopsychiatry. 2009;42(6):270-6.

33 Bus BA, Molendijk ML, Penninx BJ, Buitelaar JK, Kenis G, Prickaerts J, et al. Determinants of serum brain-derived neurotrophic factor. Psychoneuroendocrinology. 2011;36(2):228-39.

34 Bus BA, Tendolkar I, Franke B, de Graaf J, den Heijer M, Buitelaar JK, et al. Serum brain-derived neurotrophic factor: determinants and relationship with depressive symptoms in a community population of middle-aged and elderly people. World J Biol Psychiatry. 2012; 13(1):39-47.

35 Chan KL, Tong KY, Yip SP. Relationship of serum brain-derived neurotrophic factor (BDNF) and health-related lifestyle in healthy human subjects. Neurosci Lett. 2008;447(23):124-8.

36 Xu Y, Wei H, Zhu Y, Zhu Y, Zhang N, Qin J, et al. Potential serum biomarkers for the prediction of the efficacy of escitalopram for treating depression. J Affect Disord. 2019;250: $307-12$.

37 Pillai A, Buckley PF. Reliable biomarkers and predictors of schizophrenia and its treatment. Psychiatr Clin North Am. 2012;35(3):645-59.
38 Bax L, Yu LM, Ikeda N, Tsuruta H, Moons KG. Development and validation of MIX: comprehensive free software for meta-analysis of causal research data. BMC Med Res Methodol. 2006;6:50.

39 Grissom RJ, Kim JJ. Effect sizes for research: a broad practical approach. Lawrence Erlbaum Associates; 2005.

40 Moher D, Liberati A, Tetzlaff J, Altman DG. PRISMA Group. Preferred reporting items for systematic reviews and meta-analyses: the PRISMA statement. PLoS Med. 2009;6:e1000097.

41 Higgins JP, Thompson SG. Quantifying heterogeneity in a meta-analysis. Stat Med. 2002; 21(11):1539-58.

42 Benjamini Y, Hochberg Y. Controlling the false discovery rate: a practical and powerful approach to multiple testing. J R Stat Soc Series B Stat Methodol. 1995;57(1):289-300.

43 Peters JL, Sutton AJ, Jones DR, Abrams KR, Rushton L. Performance of the trim and fill method in the presence of publication bias and between-study heterogeneity. Stat Med. 2007;26(25):4544-62.

44 Aas M, Haukvik UK, Djurovic S, Bergmann $\varnothing$, Athanasiu L, Tesli MS, et al. BDNF val66met modulates the association between childhood trauma, cognitive and brain abnormalities in psychoses. Prog Neuropsychopharmacol Biol Psychiatry. 2013;46:181-8.

45 Dutt A, McDonald C, Dempster E, Prata D, Shaikh M, Williams I, et al. The effect of COMT, BDNF, 5-HTT, NRG1 and DTNBP1 genes on hippocampal and lateral ventricular volume in psychosis. Psychol Med. 2009; 39(11):1783-97.

46 Koolschijn PC, van Haren NE, Bakker SC, Hoogendoorn ML, Hulshoff Pol HE, Kahn RS. Effects of brain-derived neurotrophic factor Val66Met polymorphism on hippocampal volume change in schizophrenia. Hippocampus. 2010;20(9):1010-7.

47 Smith GN, Thornton AE, Lang DJ, Macewan GW, Ehmann TS, Kopala LC, et al. Hippocampal volume and the brain-derived neurotrophic factor Val66Met polymorphism in first episode psychosis. Schizophr Res. 2012; 134(2-3):253-9.

48 Takahashi T, Suzuki M, Tsunoda M, Kawamura Y, Takahashi N, Tsuneki H, et al. Association between the brain-derived neurotrophic factor Val66Met polymorphism and brain morphology in a Japanese sample of schizophrenia and healthy comparisons. Neurosci Lett. 2008;435(1):34-9.

49 Zugman A, Pedrini M, Gadelha A, Kempton MJ, Noto CS, Mansur RB, et al. Serum brainderived neurotrophic factor and cortical thickness are differently related in patients with schizophrenia and controls. Psychiatry Res. 2015;234(1):84-9.

50 Rizos EN, Papathanasiou M, Michalopoulou PG, Mazioti A, Douzenis A, Kastania A, et al. Association of serum BDNF levels with hippocampal volumes in first psychotic episode drug-naive schizophrenic patients. Schizophr Res. 2011;129(2-3):201-4. 
51 Rizos E, Papathanasiou MA, Michalopoulou PG, Laskos E, Mazioti A, Kastania A, et al. A longitudinal study of alterations of hippocampal volumes and serum BDNF levels in association to atypical antipsychotics in a sample of first-episode patients with schizophrenia. PLoS One. 2014;9(2):e87997.

52 Song X, Fan X, Li X, Kennedy D, Pang L, Quan M, et al. Serum levels of BDNF, folate and homocysteine: in relation to hippocampal volume and psychopathology in drug naive, first episode schizophrenia. Schizophr Res. 2014;159:51-5.

53 Song X, Quan M, Lv L, Li X, Pang L, Kennedy $\mathrm{D}$, et al. Decreased cortical thickness in drug naïve first episode schizophrenia: in relation to serum levels of BDNF. J Psychiatr Res. 2015;60:22-8.

54 Collins A, Koechlin E. Reasoning, learning, and creativity: frontal lobe function and human decision-making. PLoS Biol. 2012;10(3): e1001293.

55 Luo J, Niki K. Function of hippocampus in "insight" of problem solving. Hippocampus. 2003;13(3):316-23.

56 Zeithamova D, Schlichting ML, Preston AR. The hippocampus and inferential reasoning: building memories to navigate future decisions. Front Hum Neurosci. 2012;6:70.

57 Ahmed AO, Mantini AM, Fridberg DJ, Buckley PF. Brain-derived neurotrophic factor (BDNF) and neurocognitive deficits in people with schizophrenia: a meta-analysis. Psychiatry Res. 2015;226(1):1-13.

58 Wykes T, Huddy V, Cellard C, McGurk SR, Czobor P. A meta-analysis of cognitive remediation for schizophrenia: methodology and effect sizes. Am J Psychiatry. 2011;168(5): $472-85$.

59 Ahmed AO, Hunter KM, Goodrum NM, Batten NJ, Birgenheir D, Hardison E, et al. A randomized study of cognitive remediation for forensic and mental health patients with schizophrenia. J Psychiatr Res. 2015;68:8-18.

60 Vinogradov S, Fisher M, Holland C, Shelly W, Wolkowitz $\mathrm{O}$, Mellon SH. Is serum brain-derived neurotrophic factor a biomarker for cognitive enhancement in schizophrenia? Biol Psychiatry. 2009;66(6):549-53.

61 Penadés R, López-Vílchez I, Catalán R, Arias B, González-Rodríguez A, García-Rizo C, et al. BDNF as a marker of response to cognitive remediation in patients with schizophrenia: a randomized and controlled trial. Schizophr Res. 2018;197:458-64.

62 Keshavan MS, Eack SM, Prasad KM, Haller CS, Cho RY. Longitudinal functional brain imaging study in early course schizophrenia before and after cognitive enhancement therapy. Neuroimage. 2017;151:55-64.
63 Morimoto T, Matsuda Y, Matsuoka K, Yasuno F, Ikebuchi E, Kameda H, et al. Computerassisted cognitive remediation therapy increases hippocampal volume in patients with schizophrenia: a randomized controlled trial. BMC Psychiatry. 2018;18(1):83.

64 Matsuda Y, Makinodan M, Morimoto T, Kishimoto T. Neural changes following cognitive remediation therapy for schizophrenia: a review. Psychiatry Clin Neurosci. 2019 Nov; 73(11):676-4.

65 Ahmed AO, Bhat IA. Psychopharmacological treatment of neurocognitive deficits in people with schizophrenia: a review of old and new targets. CNS Drugs. 2014;28(4):301-18.

66 Koola MM, Praharaj SK, Pillai A. Galantamine-memantine combination as an antioxidant treatment for schizophrenia. Curr Behav Neurosci Rep. 2019;6(2):37-50.

67 Egan MF, Kojima M, Callicott JH, Goldberg TE, Kolachana BS, Bertolino A, et al. The BDNF val66met polymorphism affects activity-dependent secretion of BDNF and human memory and hippocampal function. Cell. 2003;112(2):257-69.

68 Frodl T, Amico F. Is there an association between peripheral immune markers and structural/functional neuroimaging findings? Prog Neuropsychopharmacol Biol Psychiatry. 2014;48:295-303.

69 Pillai A, Howell KR, Ahmed AO, Weinberg D, Allen KM, Bruggemann J, et al. Association of serum VEGF levels with prefrontal cortex volume in schizophrenia. Mol Psychiatry. 2016; 21(5):686-92.

70 Neugebauer K, Hammans C, Wensing T, Kumar V, Grodd W, Mevissen L, et al. Nerve growth factor serum levels are associated with regional gray matter volume differences in schizophrenia patients. Front Psychiatry. 2019;10:275.

71 Ahmed AO, Fridberg D, Hanna M, Buckley $\mathrm{PF}$. Brain-derived neurotrophic factor and neurocognitive profiles in the psychosis spectrum: findings in bipolar disorder and schizophrenia. In: Moore NB, editor. Bipolar disorders: symptoms, management, and risk factors. New York: Nova Science Publishers; 2013.

72 Farreny A, Aguado J, Corbera S, Ochoa S, Huerta-Ramos E, Usall J. Baseline predictors for success following strategy-based cognitive remediation group training in schizophrenia. J Nerv Ment Dis. 2016;204(8):585-9.

73 Gratacòs M, González JR, Mercader JM, de Cid R, Urretavizcaya M, Estivill X. Brain-derived neurotrophic factor Val66Met and psychiatric disorders: meta-analysis of case-control studies confirm association to substancerelated disorders, eating disorders, and schizophrenia. Biol Psychiatry. 2007;61(7): 911-22.
74 Hong CJ, Yu YW, Lin CH, Tsai SJ. An association study of a brain-derived neurotrophic factor Val66Met polymorphism and clozapine response of schizophrenic patients. Neurosci Lett. 2003;349(3):206-8.

75 Neves-Pereira M, Cheung JK, Pasdar A, Zhang F, Breen G, Yates P, et al. BDNF gene is a risk factor for schizophrenia in a Scottish population. Mol Psychiatry. 2005;10(2):20812.

76 Numata S, Ueno S, Iga J, Yamauchi K, Hongwei S, Ohta $\mathrm{K}$, et al. Brain-derived neurotrophic factor (BDNF) Val66Met polymorphism in schizophrenia is associated with age at onset and symptoms. Neurosci Lett. 2006; 401(1-2):1-5.

77 Rizos EN, Papadopoulou A, Laskos E, Michalopoulou PG, Kastania A, Vasilopoulos D, et al. Reduced serum BDNF levels in patients with chronic schizophrenic disorder in relapse, who were treated with typical or atypical antipsychotics. World J Biol Psychiatry. 2010;11(2 Pt 2):251-5.

78 Sanada K, Zorrilla I, Iwata Y, Bermúdez-Ampudia C, Graff-Guerrero A, Martínez-Cengotitabengoa $\mathrm{M}$, et al. The efficacy of nonpharmacological interventions on brain-derived neurotrophic factor in schizophrenia: a systematic review and meta-analysis. Int J Mol Sci. 2016;17(10):1766.

79 Shrivastava A, De Sousa A, Rao GP. Brainderived neurotrophic factor and suicide in schizophrenia: critical role of neuroprotective mechanisms as an emerging hypothesis. Indian J Psychol Med. 2016;38(6):499504

80 Terracciano A, Tanaka T, Sutin AR, Deiana B, Balaci L, Sanna S, et al. BDNF Val66Met is associated with introversion and interacts with 5-HTTLPR to influence neuroticism. Neuropsychopharmacology. 2010; 35(5): 1083-9.

81 Xia H, Zhang G, Du X, Zhang Y, Yin G, Dai J, et al. Suicide attempt, clinical correlates, and BDNF Val66Met polymorphism in chronic patients with schizophrenia. Neuropsychology. 2018 Feb;32(2):199-205.

82 Tsai SJ. Critical issues in BDNF Val66Met genetic studies of neuropsychiatric disorders. Front Mol Neurosci. 2018;11:156.

83 Naegelin Y, Dingsdale H, Säuberli K, Schädelin S, Kappos L, Barde YA. Measuring and validating the levels of brain-derived neurotrophic factor in human serum. eNeuro. 2018 Mar 22;5(2)

84 Ziegenhorn AA, Schulte-Herbrüggen $\mathrm{O}$, Danker-Hopfe H, Malbranc M, Hartung HD, Anders D, et al. Serum neurotrophins: a study on the time course and influencing factors in a large old age sample. Neurobiol Aging. 2007;28(9):1436-45. 